\title{
Potential for measurement of the tensor magnetic polarizability of the deuteron in storage ring experiments
}

\author{
Alexander J. Silenko \\ Institute of Nuclear Problems, Belarusian State University, Minsk 220030, Belarus
}

(Dated: August 15, 2021)

\begin{abstract}
General formulas describing deuteron spin dynamics in storage rings with allowance for the tensor electric and magnetic polarizabilities are derived. It is found that an initially tensor-polarized deuteron beam can acquire a final horizontal vector polarization of the order of $1 \%$. This effect allows one to measure the tensor magnetic polarizability of the deuteron in storage ring experiments. We also confirm an existence of the effect found by Baryshevsky and Gurinovich, hep-ph/0506135 and Baryshevsky, hep-ph/0510158; hep-ph/0603191 that the tensor magnetic polarizability of the deuteron causes the spin rotation with two frequencies and experiences beating for polarized deuteron beams in storage rings.
\end{abstract}

PACS numbers: 21.45.-v, 11.10.Ef, 21.10.Ky, 24.70.+s

Keywords: deuteron, tensor magnetic polarizability, tensor electric polarizability 


\section{INTRODUCTION}

Tensor electric and magnetic polarizabilities defined by spin interactions of nucleons are important parameters of the deuteron and other nuclei. In particular, measurement of tensor polarizabilities of the deuteron gives important information about spin-dependent nuclear forces.

For vector-polarized deuteron beams in storage rings, the main effects caused by the tensor polarizabilities have been investigated by Baryshevsky and Gurinovich [1] and Baryshevsky [2]. The tensor magnetic polarizability, $\beta_{T}$, produces the spin rotation with two frequencies instead of one, beating with a frequency proportional to $\beta_{T}$, and causes transitions between vector and tensor polarizations [1, 2]. We confirm the existence of these effects and carry out a detailed calculation of deuteron spin dynamics in storage rings. We use the matrix Hamiltonian obtained in Ref. [3] and derive general formulae describing the evolution of the spin. We show that an initially tensor-polarized deuteron beam can acquire a final horizontal vector polarization of the order of $1 \%$. This effect makes it possible to measure the tensor magnetic polarizability of the deuteron in storage rings.

The system of units $\hbar=c=1$ is used.

\section{HAMILTONIAN APPROACH IN THE METHOD OF SPIN AMPLITUDES}

The method of spin amplitudes uses quantum mechanics formalism to more easily describe spin dynamics (see Ref. [4]). Vector and tensor polarizations of particles and nuclei with spin $S \geq 1$ are specified by the unit polarization vector $\boldsymbol{P}$ and the polarization tensor $P_{i j}$, which are given by [5]

$$
P_{i}=\frac{<S_{i}>}{S}, \quad P_{i j}=\frac{3<S_{i} S_{j}+S_{j} S_{i}>-2 S(S+1) \delta_{i j}}{2 S(2 S-1)},
$$

where $S_{i}$ are spin matrices and $i, j=x, y, z$. Additional tensors composed of products of three or more spin matrices are needed only for the exhaustive description of polarization of particles and nuclei with spin $S \geq 3 / 2$.

The nontrivial spin dynamics predicted in Refs. [1, 2], produced by the tensor electric and magnetic polarizabilities of the deuteron, are a good example of the importance of spin-tensor interactions in the physics of polarized beams in storage rings. To describe tensor interactions of the deuteron with the method of spin amplitudes, one should use 
three-component spinors and $3 \times 3$ matrices. The method of spin amplitudes is mathematically advantageous because transporting the three-component spinor is much simpler than transporting the three-dimensional polarization vector $\boldsymbol{P}$ and five independent components of the polarization tensor $P_{i j}$ together.

We follow the traditional quantum mechanical approach [6] and use the matrix Hamilton equation and the Hamilton operator $\mathcal{H}$ for determining an evolution of the spin wave function:

$$
i \frac{d \Psi}{d t}=H \Psi, \quad \Psi=\left(\begin{array}{c}
C_{1}(t) \\
C_{0}(t) \\
C_{-1}(t)
\end{array}\right), \quad H_{i j}=<i|\mathcal{H}| j>
$$

where $H$ is $3 \times 3$ matrix, $H_{i j}$ are matrix elements of $\mathcal{H}, \Psi$ is the three-component spin wave function (spinor), with the wave function $\mid i>$ defined by $C_{i}=1$, and $i, j=1,0,-1$. In the case considered, the Hamilton operator is given by [3]

$$
\mathcal{H}=\mathcal{H}_{0}+\boldsymbol{S} \cdot \boldsymbol{\omega}_{a}-\left(\alpha_{T} \beta^{2} S_{\rho}^{2}+\beta_{T} S_{z}^{2}\right) B_{z}^{2} \gamma
$$

where $\boldsymbol{\omega}_{a}$ is the angular velocity of spin precession relative to the direction of momentum (g-2 precession), $\alpha_{T}$ is the tensor electric polarizability, $\beta=v / c$ is the normalized velocity, $B_{z}$ is the vertical magnetic field, and $\gamma$ is the Lorentz factor.

A determination of spin dynamics can be divided into several stages, namely, (i) a solution of Hamilton equation (2) and a determination of eigenvalues and eigenvectors of the matrix Hamiltonian $H$, (ii) a derivation of the spin wave function consisting in a solution of a set of three linear algebraic equations, and (iii) a calculation of the time evolution of the polarization vector and the polarization tensor.

\section{DYNAMICS OF DEUTERON SPIN IN STORAGE RINGS}

Corrections to the Hamilton operator for the deuteron polarizabilities contain scalar and tensor parts. The scalar part is spin-independent and can be disregarded. The general form of the matrix Hamiltonian $H$ has been found in Ref. [3]. When we consider the deuteron spin dynamics in a uniform magnetic field, the matrix Hamiltonian is Hermitian and takes 
the form

where [3]

$$
H=\left(\begin{array}{ccc}
E_{0}+\omega_{0}+\mathcal{A}+\mathcal{B} & 0 & \mathcal{A} \\
0 & E_{0}+2 \mathcal{A} & 0 \\
\mathcal{A} & 0 & E_{0}-\omega_{0}+\mathcal{A}+\mathcal{B}
\end{array}\right)
$$

$$
\mathcal{A}=-\frac{1}{2} \alpha_{T} B_{z}^{2} \gamma \beta^{2}, \quad \mathcal{B}=-\beta_{T} B_{z}^{2} \gamma
$$

$\omega_{0}=\left(\omega_{a}\right)_{z}$ is the angular frequency of spin rotation (g-2 frequency) and $E_{0}$ is the zero energy level. The nondiagonal components in Eq. (4) are nonresonant and can be disregarded because their average effect on the rotating spin is zero. Equation 4) shows that the deuteron spin in the horizontal plane is affected not only by the tensor magnetic polarizability but also by the tensor electric one.

It can be easily checked that matrix Hamiltonian (44) coincides with Hamilton operator (3) expressed in matrix form. The coincidence of Eqs. (3) and (44) results from the fact that the considered Hamilton operator is independent of coordinates.

Calculations of spin dynamics defined by Eqs. (44),(5) are much simpler than the calculations fulfilled in Ref. [3] because of the absence of resonance effects. The connection between spin amplitudes and components of polarization vector and polarization tensor is given by Eq. (44) in Ref. [3]. If the deuteron beam is vector-polarized and the direction of its polarization is characterized by the spherical angles $\theta$ and $\psi$, the general equation defining the evolution of deuteron polarization has the form

$$
\begin{gathered}
P_{\rho}(t)=\sin \theta \cos \left(\omega_{0} t+\psi\right) \cos (b t) \\
-\sin \theta \cos \theta \sin \left(\omega_{0} t+\psi\right) \sin (b t), \\
P_{\phi}(t)=\sin \theta \sin \left(\omega_{0} t+\psi\right) \cos (b t) \\
+\sin \theta \cos \theta \cos \left(\omega_{0} t+\psi\right) \sin (b t), \quad P_{z}(t)=P_{z}(0),
\end{gathered}
$$

where

$$
b=\mathcal{B}-\mathcal{A}=-\left(\beta_{T}-\frac{1}{2} \alpha_{T} \beta^{2}\right) B_{z}^{2} \gamma
$$

The initial vector polarization is given by

$$
\boldsymbol{P}(0)=\sin \theta \cos \psi \boldsymbol{e}_{\rho}+\sin \theta \sin \psi \boldsymbol{e}_{\phi}+\cos \theta \boldsymbol{e}_{z}
$$

Eqs. (6), (77) show that the tensor polarizabilities cause the spin rotation of the vectorpolarized deuteron beam with two frequencies $\omega_{0} \pm b$ instead of $\omega_{0}$ and therefore experience 
beating with the frequency $\Delta \omega=-2 b$. Thus, these equations confirm the conclusion given by Baryshevsky and Gurinovich [1] and Baryshevsky [2]. Eq. (77) displays that the spin rotation is also affected by the tensor electric polarizability. Although the effect predicted in Refs. [1, 2] is not negligible, its direct observation is very difficult. There are three independent theoretical predictions for the value of the tensor electric polarizability of the deuteron, namely $\alpha_{T}=-6.2 \times 10^{-41} \mathrm{~cm}^{3}$ [7], $-6.8 \times 10^{-41} \mathrm{~cm}^{3}$ [8], and $3.2 \times 10^{-41} \mathrm{~cm}^{3}$ [9]. The first two values are very close to each other but they do not agree with the last result. The theoretical estimate for the tensor magnetic polarizability of deuteron is $\beta_{T}=$ $1.95 \times 10^{-40} \mathrm{~cm}^{3}[7,8]$. The duration of measurement $t$ is restricted by the spin coherence time $\tau$. If we base our estimate on the values corresponding to the planned deuteron electricdipole-moment experiment in storage rings $[10](\gamma=1.28, \beta=0.625, \tau \sim 1000 \mathrm{~s}$, and $B_{z}=3 \mathrm{~T}$ ), then $b \sim 10^{-5} \mathrm{~s}^{-1}$ and $b t \lesssim 10^{-2}$. In this case, systematical errors caused by betatron oscillations, field defects, and misalignments of magnets can appreciably exceed small perturbations of spin rotation produced by the deuteron tensor polarizabilities.

We propose a significant improvement in the precision of a possible experiment. Measurement of the effect can be strongly simplified with the use of a tensor-polarized deuteron beam. If the initial vector polarization of such a beam is zero, any interactions of the magnetic moment of deuteron with external fields cannot lead to the appearance of vector polarization. Therefore, nonzero vector polarization of the beam can be produced by nothing but the tensor interactions. The initial tensor polarization can correspond to a zero projection of the deuteron spin onto the preferential direction. When this direction is defined by the spherical angles $\theta$ and $\psi$, the initial polarization is given by

$$
\begin{gathered}
\boldsymbol{P}(0)=0, \quad P_{\rho \rho}(0)=1-3 \sin ^{2} \theta \cos ^{2} \psi \\
P_{\phi \phi}(0)=1-3 \sin ^{2} \theta \sin ^{2} \psi, \quad P_{z z}(0)=1-3 \cos ^{2} \theta, \\
P_{\rho \phi}(0)=-\frac{3}{2} \sin ^{2} \theta \sin (2 \psi), \\
P_{\rho z}(0)=-\frac{3}{2} \sin (2 \theta) \cos \psi, \quad P_{\phi z}(0)=-\frac{3}{2} \sin (2 \theta) \sin \psi
\end{gathered}
$$

In this case, the time dependence of the polarization vector has the form

$$
\begin{gathered}
P_{\rho}(t)=\sin (2 \theta) \sin \left(\omega_{0} t+\psi\right) \sin (b t), \\
P_{\phi}(t)=-\sin (2 \theta) \cos \left(\omega_{0} t+\psi\right) \sin (b t), \quad P_{z}(t)=0 .
\end{gathered}
$$

The final vector polarization is horizontal. 
Spin dynamics can be easily calculated for any other initial tensor polarization of the deuteron beam.

Eq. (9) shows the possibility of measurement of the quantity $b$ in storage ring experiments. The final vector polarization of the beam is of the order of $1 \%$.

\section{DISCUSSION AND SUMMARY}

The analysis presented confirms the results obtained by Baryshevsky and Gurinovich [1] and Baryshevsky [2]. It is shown that the predicted rotation of the deuteron spin with two frequencies, beating, and transitions between vector and tensor polarizations in a uniform magnetic field are produced not only by the tensor magnetic polarizability but also by the tensor electric polarizability. Nevertheless, the latter quantity gives a minor contribution to the effect. If experimental conditions correspond to the planned deuteron electric-dipolemoment experiment in storage rings [10] $\left(\beta^{2}=0.4\right)$ and theoretical estimates for the tensor polarizabilities of the deuteron given in Refs. [7, 8, 9] are used, the expected relative importance of $\beta_{T}$ is one order of magnitude greater.

Unfortunately, the expected spin coherence time (about $1000 \mathrm{~s}$ [10]) is too short to register beating. In this case, systematical errors can prevent the observation of small perturbations of spin rotation conditioned by the tensor polarizabilities of the deuteron. Possibly, the effect predicted in Refs. [1, 2] may be discovered with the use of a Penning trap used as a minicyclotron, since the Penning trap provides a much longer duration of measurement.

The precision of a possible storage ring experiment can be significantly improved, if the deuteron beam is tensor-polarized. If the initial vector polarization of such a beam is zero, any interactions linear in the spin cannot lead to the appearance of vector polarization. The final vector polarization also cannot result from the betatron oscillations, field defects, misalignments of magnets and other potential sources of systematical errors. Therefore, the tensor interaction of spin with the magnetic field defined by Eqs. (44),(15), and (7)-(9) is the only reason for nonzero vector polarization of the beam. Eq. (9) shows that the beam can acquire the final horizontal vector polarization of the order of $1 \%$. Known experimental methods [11] permit safe measurement of such a polarization and therefore a determination of the tensor magnetic polarizability of the deuteron. In addition, the observation of the predicted effect would prove the importance of taking into account spin-tensor interactions 
in storage ring physics.

The rotation of the polarization vector in the horizontal plane complicates a measurement of beam polarization. Two possible solutions of this problem are to eliminate the $\mathrm{g}-2 \mathrm{spin}$ rotation with an additional radial electric field (see Ref. [12]) or to use Siberian snakes and a stimulation of spin resonance [1, 2]. Both of these methods need additional devices (electric field plates or resonators) and result in a significant complication of an experimental setup. We propose direct measurement of the beam polarization in a fixed direction. The availability of the proposed method is based on the attainability of the spin coherence time about 1000 s. In this case, one holds the predicted polarization of the beam until the appearance of a measurable vector polarization. Spin coherence can be maintained because of the independence of the $\mathrm{g}-2$ frequency on the deuteron momentum for an appropriate length of straight ring sections [10]. The spin coherence may also be supported by a small deuteron momentum spread $\Delta p / p$. It is important that the ratio of the $\mathrm{g}-2$ frequency to the cyclotron one is small enough $\left[\omega_{0} / \omega_{c}=\gamma(g-2) / 2=-0.143 \gamma\right.$ in semicircular sections]. Under these conditions, the spin evolution is clearly defined and one can choose an appropriate measurement time interval. The experiment can be implemented in one of the existing storage rings.

Since Eq. (7) contains both tensor polarizabilities, the tensor electric polarizability of the deuteron can also be measured in the proposed experiment and the obtained precision of polarization measurements [11] should be significantly improved. The experiment should be performed with different values of the beam momentum (and the vertical magnetic field) on the same ring. In this case, both tensor polarizabilities of the deuteron can be determined. Another method of determination of the deuteron's tensor electric polarizability has been

proposed in Ref. [3]. The use of the Penning trap in experiments with tensor-polarized deuterons can also be helpful for measuring the tensor polarizabilities.

\section{Acknowledgements}

The author is grateful to V.G. Baryshevsky for bringing the considered problem to his attention and for helpful discussions and comments. This work was supported by the Be- 
larusian Republican Foundation for Fundamental Research.

[1] V. G. Baryshevsky, A. A. Gurinovich, hep-ph/0506135.

[2] V. G. Baryshevsky, hep-ph/0510158; hep-ph/0603191.

[3] A. J. Silenko, Phys. Rev. C 75, 014003 (2007).

[4] E. D. Courant, Bull. Am. Phys. Soc. 7, 33 (1962); E. D. Courant and R. D. Ruth, BNL Report No. 51270 (1980); M. G. Minty, F. Zimmermann, Measurement and Control of Charged Particle Beams (Springer-Verlag, Berlin, 2003), Chap. 10.

[5] S. R. Mane, Yu. M. Shatunov and K. Yokoya, Rep. Prog. Phys. 68, 1997 (2005).

[6] R. P. Feynman, R. B. Leighton, M. Sands, The Feynman Lectures on Physics. V. 2 (AddisonWesley, Reading, MA, 1964).

[7] J.-W. Chen, H. W. Grießhammer, M. J. Savage, R. P. Springer, Nucl. Phys. A 644, 221 (1998).

[8] X. Ji, Y. Li, Phys. Lett. B 591, 76 (2004).

[9] J. L. Friar and G. L. Payne, Phys. Rev. C 72, 014004 (2005).

[10] Y. F. Orlov, W. M. Morse, and Y. K. Semertzidis, Phys. Rev. Lett. 96, 214802 (2006).

[11] L.S. Azhgirey et al., Pisma Fiz. Elem. Chast. Atom. Yadra 2, 91 (2005) [Phys. Part. Nucl. Lett. 2,122 (2005)]; D. Chiladze et al., Phys. Rev. ST Accel. Beams 9, 050101 (2006).

[12] F. J. M. Farley, K. Jungmann, J. P. Miller, W. M. Morse, Y. F. Orlov, B. L. Roberts, Y. K. Semertzidis, A. Silenko, and E. J. Stephenson, Phys. Rev. Lett. 93, 052001 (2004). 